\title{
Compliance with antibiotic prophylaxis in children with sickle cell anemia: a prospective study
}

\author{
Enio Latini Bitarães, ${ }^{1}$ Benigna Maria de Oliveira, ${ }^{2}$ Marcos Borato Viana ${ }^{3}$
}

\begin{abstract}
Objective: To prospectively assess compliance with antibiotic prophylaxis among children with sickle cell anemia.

Methods: A total of 108 children (aged 3 months to $4 \frac{1}{2}$ years, $45 \%$ male) were recruited from the Hematology Center in Belo Horizonte, Brazil, and followed up for 15 months. Data on compliance were obtained from three interviews with the primary caregivers, from the children's medical records and from assay of antibacterial activity in urine samples of 81 children. Antibiotics were available free of charge.

Results: Penicillin was used in 106 cases (majority by oral route); erythromycin in 2 cases. Urine samples tested positive for the antibiotic in $56 \%$ of the cases; $48 \%$ of the caregivers assured during interviews that all doses had been administered to children; $89 \%$ of medical files recorded no compliance failures. Considering a child compliant if none or just one of these methods detected missing doses, the rate of compliance was $67 \%$. The agreement between methods for assigning a child as compliant or not was low. No significant association of compliance rate with gender, nutritional status, per capita income of the family, caregivers' schooling, or number of family members was demonstrated.

Conclusions: Compliance rate with prophylactic antibiotic therapy was low when assessed by interviews and urine tests and overestimated by analysis of medical records. Compliance should preferably be assessed by several methods due to the complex character of its measurement. The results of the present study suggest the need for a comprehensive educational program involving healthcare professionals, families and children with sickle cell anemia.

J Pediatr (Rio J). 2008;84(4):316-322: Sickle cell anemia, antibiotic prophylaxis, treatment refusal, guideline adherence, penicillin $\mathrm{V}$.
\end{abstract}

\section{Introduction}

Sickle cell anemia (SCA) is an inherited blood disorder for which the optimal treatment remains uncertain. Therefore, approaches to reduce morbidity and mortality should rely upon the early diagnosis of SCA through newborn screening, prevention of complications, and patient care delivered by a multiprofessional team. ${ }^{1}$ Severe infections, such as pneumonia, sepsis and meningitis, are the most common causes of death among children with SCA younger than 5 years of age. Starting at age 3-4 months through 5 years, penicillin is administered as a preventive measure to ward off these infections caused mainly by Streptococcus pneumoniae. ${ }^{2-4}$
The compliance of parents or caregivers with prophylactic antibiotic therapy prescribed for their children is a challenge and healthcare professionals must better understand the process of compliance considering it as an integral part of treatment plan. ${ }^{5-7}$

Several deaths, suffering, need for hospitalization and unnecessary medical care expenditures could be avoided if healthcare professionals were better informed and had the means to identify noncompliance with antibiotic prophylaxis as the cause of severe infections in children with SCA.

Compliance with treatment cannot be seen as a simple process that starts with a prescription from the healthcare pro-

1. Mestre. Enfermeiro. Preceptor, Fundação Mineira de Educação e Cultura - Faculdade de Ciências da Saúde (FUMEC-FCS), Belo Horizonte, MG, Brazil.

2. Doutora. Professora adjunta, Departamento de Pediatria, Faculdade de Medicina, Universidade Federal de Minas Gerais (UFMG), Belo Horizonte, MG, Brazil.

3. Doutor. Professor titular, Departamento de Pediatria, Faculdade de Medicina, UFMG, Belo Horizonte, MG, Brazil.

No conflicts of interest declared concerning the publication of this article.

Suggested citation: Bitarães EL, de Oliveira BM, Viana MB. Compliance with antibiotic prophylaxis in children with sickle cell anemia: a prospective study. J Pediatr (Rio J). 2008;84(4):316-322.

Manuscript received Apr 14 2008, accepted for publication May 282008.

doi:10.2223/JPED. 1819 
fessional and ends with the patient's and/or caregivers' agreement with the prescribed medication. Several socioeconomic, cultural and behavioral factors have a strong influence on this process, what makes compliance difficult to measure only through objective methods. ${ }^{8,9}$

The purpose of this study was to assess compliance with prophylactic antibiotic therapy among children with SCA.

\section{Methods}

We obtained a convenience sample of children with SCA (HbSS) diagnosed through a state-administered screening program (Programa Estadual de Triagem Neonatal de Minas Gerais, PETN-MG), followed at the Hematology Center in Belo Horizonte (HBH), Brazil, aged 3-54 months, on antibiotic prophylaxis (oral penicillin $\mathrm{V}$, benzathine penicillin or erythromycin) in accordance with the Fundação Hemominas protocol. Families were recruited between January and April 2005 by the time of the child's scheduled follow-up appointment. A total of 125 families were invited to participate in this prospective study corresponding to 128 children (three families with two siblings); one family refused to sign the informed consent. This sample represented about $2 / 3$ of all children with SCA eligible for the study; the remaining children did not have a scheduled follow-up appointment during the recruitment period or did not report to the appointment. Of the 127 children enrolled in the study, 19 were subsequently excluded: 12 because of loss to follow up, two because they were transferred to another health unit, one because diagnosis was $\mathrm{S} \beta^{0}$-thalassemia, and four died in the beginning of the study; leaving 108 eligible children for analysis.

Three methods were used to assess compliance: standardized interview questionnaires, information abstracted from medical records and urine assay for antibiotic activity.

A questionnaire was administered to each primary caregiver by the time of the follow-up appointments, in three different occasions: during the recruitment period, on an intermediate date and the last one at the end of the study. One of the researchers (ELB) performed $70 \%$ of the interviews, and the remaining 30\% were performed by two undergraduate nursing students trained by him. Appointment intervals ranged from 2 to 6 months, depending on the routine scheduling system and the family compliance rates for follow-up. To enable all families to have three interviews, the total study period comprised the 15 months from January 2005 to April 2006. During the interviews, the caregivers were questioned about the child's ongoing medication at the time of application of the questionnaire; dosage frequency; person responsible for each dose administration; dosage schedule and intervals; number of missed doses and reason for missing doses; medication administration difficulties; understanding of medical instructions; and whether the caregiver had been instructed by other healthcare professionals on medication administration. Antibiotics were available free of charge for all patients.

Assessment of compliance by medical records abstracting was performed simultaneously to the application of the questionnaires and focused on the answers the caregivers gave the physician to the questions regarding regular, irregular or no use of the medication.

To assess compliance by urine assay for antibiotic activity, each caregiver was asked to provide a urine sample of the child during the study period. Children had urine collected following the application of the questionnaire when caregivers informed administration of oral penicillin in that morning or administration of benzathine penicillin 1-7 days prior to the scheduled appointment at the hematology center. Several caregivers informed that they had not administered oral penicillin to the child that morning due to preparations for visiting the hematology center. In these cases, at the end of the interview, caregivers were given the necessary orientation and a mail-in kit containing material for filter-paper collection of urine samples at home. Time for urine collection was established in written form, according to the information obtained from the questionnaire on the administration of the oral antibiotic, without letting the caregiver draw out the connection between antibiotic administration and time of urine collection. We requested the children who had achieved sphincter control to collect urine 1-2 $\mathrm{h}$ after the usual time of antibiotic administration informed by the mother. Children lacking sphincter control were given a urine collector which should be used 30 min after the usual time of antibiotic administration and kept for $1.5 \mathrm{~h}$. Collection should be performed daily until the adequate sample of urine was obtained as previously oriented. Children on intramuscular penicillin, who would collect urine sample at home, should observe the urine collection schedule at the hematology center. All material necessary to the collection, such as self-addressed stamped envelopes, small protective envelope containing a filter-paper strip marked at one end (to dip into urine), urine collectors, was available for the caregivers.

No special care was given to the storage of the envelopes received via mail. However, urine samples were assayed for antibiotic activity only when delivered within 7 days of the mailing date.

The actual purpose of urine collection was not revealed to the caregivers to avoid interference in the process of compliance gaining. Patients were informed that it was a routine urine examination.

Each urine sample was assayed by an established method (bacteria highly sensitive to penicillin and erythromycin with agar disk diffusion). Tests were considered positive when an inhibition halo was visible around the filter-paper strip wetted in urine. ${ }^{10-12}$ 
Data on family history and socioeconomic situation were obtained from the children's medical records. We analyzed data regarding family income, number of family members and caregiver's schooling.

To assess nutritional status, indicators were height-for-age (HAZ) and weight-for-height (WHZ) expressed as z scores, considering weight and height of the child at study enrollment. Reference population was set as standardized by the National Center for Health Statistics (CDC, 2000). ${ }^{13}$

To analyze compliance failures, frequency tables were constructed to characterize distribution of categorical variables. For analysis, each child was considered as an individual case, regardless of kinship (three pairs of siblings). Fisher exact test was used to explore associations between these variables ( 2 $\times 2$ tables) and chi-square trend analysis was used for $3 \times 3$ tables when variables showed degree of order, such as number of family members, caregiver's schooling, etc. Mann-Whitney nonparametric test was used to compare groups with good and poor compliance rates in relation to the variable showing a non-normal distribution by Kolmogorov-Smirnov test, such as age and per capita income of the family. Similarly, Kruskal-Wallis nonparametric test was used when the variable for compliance had three levels, such as the number of times patient failed to take the antibiotic dose.

Final compliance was assessed by means of a variable which involved the three methods utilized. Children were considered compliant if compliance was achieved through the three methods applied or if only one of the methods exhibited compliance failures. Otherwise they were considered non-compliant, i.e., when noncompliance evidence was found in two or all three methods. A value of $p<0.05$ was considered statistically significant.

Parents/guardians of the children signed an informed consent for enrollment in the study. It had been previously approved by the Research Ethics Committee of the Fundação Hemominas on Nov 09, 2004 (protocol no. 116).

\section{Results}

Of the 108 children studied, 49 were male (45.4\%) and 59 were female (54.6\%). Mean age was 25.3 months at the first interview. Per capita income of the family ranged from 0 to 3.06 of a monthly minimum wage (percentile 25: 0.23 ; mean: 0.33; percentile 75: 0.53).

Thirty-four families had two or three members (31.5\%), 45 had four or five members ( $41.6 \%$ ) and 29 had six or more members $(26.9 \%)$. The mother was the primary caregiver in 102 of the cases $(94.4 \%)$.

Regarding caregiver's schooling, 65 had not completed elementary school (60\%), 25 had either completed elementary school or not completed high school (23\%) and 16 had

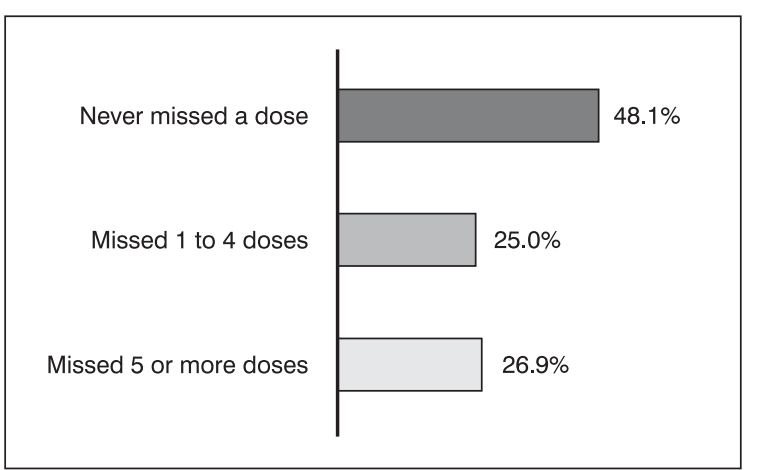

Figure 1 - Regularity of prophylactic antibiotic administration, according to questionnaires applied to 108 caregivers of children with sickle cell anemia

completed high school (15\%). Only one caregiver was illiterate and another one had a college degree.

At first interview, 26 children (24.1\%) were considered malnourished by the $\mathrm{WHZ}$ anthropometric indicator and $13.9 \%$ by the HAZ indicator.

Erythromycin was prescribed in two cases (2\%), benzathine penicillin in 11 cases ( $10 \%)$, oral penicillin $V$ in 82 cases (76\%) and alternating with benzathine in the remaining 13 cases (12\%).

Figure 1 shows the results when, considering the three interviews applied, the caregivers were questioned about the number of times they had failed to administer the antibiotic. More than half of all caregivers (52\%) admitted having forgotten, at least once, to administer an antibiotic dose.

With respect to knowledge regarding the child's medication therapy, inaccurate answers were observed in 13 interviews; 11 caregivers could not correctly say the word "penicillin," nor a similar word which could resemble penicillin, and two caregivers completely ignored that the child was on antibiotic therapy. Concerning understanding of medical instructions on antibiotic prophylaxis, only three caregivers reported lack of understanding during the first interviews, with subsequent improvement in understanding.

When questioned about whether another healthcare professional, in addition to their physician, had provided them with further orientation on penicillin administration, reference to nurses performing this task was observed in the interviews of nine children only.

In 96 children (89\%), no compliance failures in antibiotic administration occurred according to information abstracted from the medical records retrospectively analyzed.

Of the 108 children studied, 81 provided urine samples for antibiotic activity testing: 36 (44\%) tested negative and $45(56 \%)$ tested positive for presence of antibiotic. Of the 81 samples, only 11 were collected right after the interview, when the mother informed administration of oral penicillin in that 
Table 1 - Association between compliance failures detected by interviews and assay of antibacterial activity in urine samples of 81 children with sickle cell disease on antibiotic prophylaxis*

Compliance rate by questionnaires (number of children and $\%)^{+}$

\begin{tabular}{|c|c|c|c|c|}
\hline Antibiotic in urine ${ }^{*}$ & 0 & 1 & 2 & Total \\
\hline No & $13(34.2 \%)$ & $13(59.1 \%)$ & $10(47.6 \%)$ & $36(44.4 \%)$ \\
\hline Yes & $25(65.8 \%)$ & $9(40.9 \%)$ & $11(52.4 \%)$ & $45(55.6 \%)$ \\
\hline Total & $38(100 \%)$ & $22(100 \%)$ & $21(100 \%)$ & $81(100 \%)$ \\
\hline
\end{tabular}

morning or administration of benzathine penicillin within the last 7 days. Five of these samples tested positive (45.5\%). Of the 70 mail-in samples, 40 tested positive (57\%).

Considering the child compliant if none or only one of the methods applied detected compliance failures, compliance rate reached $67 \%$ (72/108). However, the level of concordance between the three methods used to measure compliance was low (Tables 1-3).

No significant association was found between compliance and caregivers' schooling, children nutritional status, gender, age, number of family members and per capita income of the family.

\section{Discussion}

Compliance is a multifaceted process involving patients' attitude towards their treatment and may also be influenced by the measurement method of choice, location where the study is carried out and the definition of "good compliance" adopted. $^{5}$

The number of children included in this study $(n=108)$ was higher than that of most studies with similar purposes. ${ }^{14-19}$ All children were recruited from the PETN-MG and followed up at the $\mathrm{HBH}$, allowing for sample homogeneity.

Application of questionnaires to the caregivers is a method commonly used in clinical practice and easy to perform. Questionnaires might not be the most accurate tool to assess compliance, but they proved helpful in learning about patterns of compliance failures and are useful as a means of starting a conversation about the theme with the caregiver, ${ }^{20}$ as conducted in the present study. Due to its "investigative" character, this method may overestimate compliance rates, what might have happened in the present study as well as observed in a Brazilian study on compliance among children with asthma. ${ }^{21}$

Table 2 - Association between compliance failures detected by analysis of medical records and assay of antibacterial activity in urine samples of 81 children with sickle cell disease on antibiotic prophylaxis*

Compliance rate by medical records

(number of children and \%) ${ }^{+}$

Antibiotic in urine *

Yes

Total
0

$33(45.8 \%)$

$39(54.2 \%)$

$72(100 \%)$
1

Total

$\begin{array}{ll}3(33.3 \%) & 36(44.4 \%) \\ 6(66.7 \%) & 45(55.6 \%) \\ 9(100 \%) & 81(100 \%)\end{array}$

\section{$* \mathrm{p}=0.72$.}

+ Compliance rate by medical records = compliance failures detected through information from the patients' medical records: $0=$ used antibiotic, accord-

ing to notes in medical records; 1 = did not use or used on an irregular basis, according to, at least, one of the notes.

* Antibiotic in urine = compliance failures detected by urine assay for antibiotic activity; no: tested negative; yes: tested positive. 
Table 3 - Association between compliance failures detected by interviews and analysis of medical records of 108 children with sickle cell disease on antibiotic prophylaxis*

Compliance rate by questionnaires (number of children and $\%)^{+}$

\begin{tabular}{|c|c|c|c|c|}
\hline $\begin{array}{l}\text { Compliance by } \\
\text { medical records }\end{array}$ & 0 & 1 & 2 & Total \\
\hline 0 & $48(92.3 \%)$ & $25(92.6 \%)$ & $23(79.3 \%)$ & $96(88.9 \%)$ \\
\hline 1 & $4(7.7 \%)$ & $2(7.4 \%)$ & $6(20.7 \%)$ & $12(11.1 \%)$ \\
\hline Total & $52(100 \%)$ & $27(100 \%)$ & $29(100 \%)$ & $108(100 \%)$ \\
\hline
\end{tabular}

$* \mathrm{p}=0.098$ (chi-square trend analysis).

+ Compliance rate by questionnaires = total number of times in which patient did not take the antibiotic although no medical orientation for weaning treatment: $0=$ none, $1=$ one to four times and 2 = five or more times.

${ }^{\ddagger}$ Compliance by medical records $=$ compliance failures detected through information from the patients' medical records: $0=$ used antibiotic, according to notes in medical records; 1 = did not use or used on an irregular basis, according to, at least, one of the notes.

Forgetfulness was the main reason reported by caregivers for missing the antibiotic doses, as previously evidenced in studies carried out by the authors on children with lymphoblastic leukaemia. ${ }^{22,23}$ Most of the caregivers reported good understanding of the information delivered by the doctors on the child's health condition. However, the results revealed that caregivers were more concerned with disease-related issues than with antibiotic prophylaxis.

The analysis of the medical records revealed that almost $90 \%$ of the children would be using prophylactic antibiotics on a regular basis, strongly contrasting with data obtained from the interviews and urine assay for antibiotic activity. This finding corroborates the opinion that estimates carried out by the doctor in charge should be considered an inaccurate method to assess compliance. ${ }^{21,24}$

Negative results accounted for $44 \%$ of the urine tests for antibiotic activity, similar to results found by other authors. ${ }^{44,15}$ The mail-in technique for filter-paper collection of urine samples at home proved to be a practical and feasible method to assess compliance with antibiotic therapy in a long-term study predicting collection of several urine samples of each patient.

Divergence of results between the three methods applied in this study has also been observed by other authors. ${ }^{15,17}$

In accordance with the literature, no significant association was found between compliance and gender, caregiver's schooling, number of family members, nutritional status or per capita income of the family. In Brazil, in children with lymphoblastic leukaemia, a statistically significant association was observed between compliance and malnutrition, probably due to socioeconomic condition. ${ }^{22}$

These findings confirm that assessment of compliance is a complex process, making compliance difficult to measure accurately through both direct and indirect methods. Among the direct methods, we quote the direct observation of patient taking the medication, not feasible on a daily routine, and assay of blood or urine levels of the medication in use or of an associated biological marker. Among the indirect methods, we quote the questionnaires and interviews, manual or electronic tablet count, pharmacy registration on dispensed drugs, assessment of clinical response or physiological effects of the drug, and patient diary. ${ }^{25}$ The exclusive use of objective methods is not enough to widely approach the problem. Several professionals' and researchers' main concerns lie on the feasibility of measuring the extent of compliance by means of methods considered objective and, then, associate the degree of compliance with factors that might facilitate or hinder compliance gaining. This approach to compliance, also adopted in the present study, tends to disregard important aspects, such as the several information sources available for patients, information provided by healthcare professionals, influence of the media and of family behavior, which make compliance an active and intentional process. ${ }^{4,26-28}$

Finally, an important question that stems from this scenario is: what would be the adequate level of compliance with penicillin therapy to achieve adequate prevention of severe infections in children with SCA? The literature does not provide, as yet, a clear answer to this question, neither to the SCA case, neither to most of diseases for which antibiotic prophylaxis is an indication. Consumption of more than $33 \%$ of penicillin reduced the risk of recurrent rheumatic fever, and usage of $88 \%$ of antihypertensive drugs was enough to maintain blood pressure under control. ${ }^{20}$ The methodology employed in the present study did not allow for approaching this aspect, since there would be a need for a systematic recording of the infection episodes, their severity and probable etiology, and also methods and techniques to assess compliance in a continuous scale or, at least, in several increasing 
or decreasing categories which could reflect the degree of compliance.

Preference for using intramuscular benzathine penicillin every 3 weeks instead of daily administration of oral penicillin comes as an alternative which could, apparently, improve treatment compliance. Due to the limited number of children in the present study that used benzathine penicillin, a valid comparison was not possible. This alternative is many times rejected by the children and their parents because it is painful; additionally, this alternative would require empirical proof of greater compliance rates than those of oral administration in children with SCA, who experience on a regular basis SCA-related vaso-occlusive painful episodes.

In conclusion, the "noncompliance" problem remains a challenge for healthcare professionals. According to the literature and to the results of our study, as yet, no method has proved completely reliable to assess compliance. However, investigation of compliance, in light of direct and/or indirect methods, as the one carried out in the present study, is certainly a valid approach. The work that we developed contributed to improving knowledge regarding the population studied and their behavior and also regarding specifics on the assistance provided by the institution. Information obtained on several aspects regarding compliance in a group of patients with a chronic and hereditary disease may benefit patients and their families, as well as contribute towards the education of the health team involved in patient care. Establishment of new protocols for patient care, improvement of doctor/family/child relationship, systemization of the assistance by nurses, social assistants and other healthcare professionals to parents, caregivers and the children's teachers are actions that will assure a better quality of attention to the children with SCA.

\section{Acknowledgments}

We would like to thank the administrative staff and healthcare professionals at the ambulatory care of Fundação Hemominas/HBH for their assistance. A special thanks to the UFMG undergraduate students Ed Wilson, Fabiano Wendel, Marcos Vinicius Figueiredo and Stefânia Barros and to Dra. Mitiko Murao, for her great assistance during several stages of the study.

\section{References}

1. Agência Nacional de Vigilância Sanitária (ANVISA). Manual de diagnóstico e tratamento de doenças falciformes. Brasília, DF: Ministério da Saúde; 2002.

2. Zarkowsky HS, Gallagher D, Gill FM, Wang WC, Falletta JM, Lande WM, et al. Bacteremia in sickle hemoglobinopathies. J Pediatr. 1986;109:579-85.

3. Gaston MH, Verter JI, Woods G, Pegelow C, Kelleher J, Presbury G, et al. Prophylaxis with oral penicillin in children with sickle cell anemia. A randomized trial. N Engl J Med. 1986;314: 1593-9.
4. Pegelow $\mathrm{CH}$, Armstrong FD, Light S, Toledano SR, Davis J. Experience with the use of prophylactic penicillin in children with sickle cell anemia. J Pediatr. 1991;118:736-8.

5. Matsui DM. Drug compliance in pediatrics. Clinical and research issues. Pediatr Clin North Am. 1997;44:1-14.

6 . Wright EC. Non-compliance-or how many aunts has Matilda? Lancet. 1993;342:909-13.

7. Day S, Brunson G, Wang W. A successful education program for parents of infants with newly diagnosed sickle cell disease. J Pediatr Nurs. 1992; 7:52-7.

8. La Greca AM. Issues in adherence with pediatric regimens. J Pediatr Psychol. 1990;15:423-36.

9. Conrad P. The meaning of medications: another look at compliance. Soc Sci Med. 1985;20:29-37.

10. Charney E, Bynum R, Eldredge D, Frank D, MacWhinney JB, McNabb N, et al. How well do patients take oral penicillin? A collaborative study in private practice. Pediatrics. 1967;40: 188-95.

11. Bergman $A B$, Werner RJ. Failure of children to receive penicillin by mouth. N Engl J Med. 1963;268:1334-8.

12. Markowitz M, Gordis L. A mail-in technique for detecting penicillin in urine: application to the study of maintenance of prophylaxis in rheumatic fever patients. Pediatrics. 1968;41:151-3.

13. Dean AG, Arner TG, Sunki GG, Friedman R, Lantinga M, Sangam $S$, et al. Epi InfoTM. A database and statistics program for public health professionals. Atlanta, GA: Centers for Disease Control and Prevention; 2002.

14. Teach SJ, Lillis KA, Grossi M. Compliance with penicillin prophylaxis in patients with sickle cell disease. Arch Pediatr Adolesc Med. 1998;152:274-8.

15. Cummins D, Heuschkel R, Davies SC. Penicillin prophylaxis in children with sickle cell disease in Brent. BMJ. 1991;302: 989-90.

16. Berkovitch M, Papadouris D, Shaw D, Onuaha N, Dias C, Olivieri NF. Trying to improve compliance with prophylactic penicillin therapy in children with sickle cell disease. $\mathrm{Br}$ J Clin Pharmacol. 1998;45:605-7.

17. Elliott V, Morgan S, Day S, Mollerup LS, Wang W. Parental health beliefs and compliance with prophylactic penicillin administration in children with sickle cell disease. J Pediatr Hematol Oncol. 2001; 23:112-6.

18. Buchanan GR, Siegel JD, Smith SJ, De Passe BM. Oral penicillin prophylaxis in children with impaired splenic function: a study of compliance. Pediatrics. 1982;70:926-30.

19. Babiker MA. Compliance with penicillin prophylaxis by children with impaired splenic function. Trop Geogr Med. 1986;38: 119-22.

20. Steiner JF, Earnest MA. The language of medication-taking. Ann Intern Med. 2000;132:926-30.

21. Lasmar LM, Camargos PA, Costa LF, Fonseca MT, Fontes MJ, Ibiapina CC, et al. Compliance with inhaled corticosteroid treatment: rates reported by guardians and measured by the pharmacy. J Pediatr (Rio J). 2007;83:471-6.

22. de Oliveira BM, Viana MB, Zani CL, Romanha AJ. Clinical and laboratory evaluation of compliance in acute lymphoblastic leukaemia. Arch Dis Child. 2004;89:785-8.

23. de Oliveira BM, Viana MB, Arruda LM, Ybarra MI, Romanha AJ. Avaliação da adesão ao tratamento através de questionários: estudo prospectivo de 73 crianças portadoras de leucemia linfoblástica aguda. J Pediatr (Rio J). 2005;81:245-50. 
24. Tebbi CK. Treatment compliance in childhood and adolescence. Cancer. 1993;71:3441-9.

25. Osterberg L, Blaschke T. Adherence to medication. N Engl J Med. 2005;353:487-97.

26. Leite SN, Vasconcellos MP. Adesão à terapêutica medicamentosa: elementos para a discussão de conceitos e pressupostos adotados na literatura. Cienc Saude Coletiva. 2003;8:775-82.

27. Shope JT. Medication compliance. Pediatr Clin North Am. 1981; 28:5-21.

28. Di Matteo MR. The role of effective communication with children and their families in fostering adherence to pediatric regimens. Patient Educ Couns. 2004;55:339-44.
Correspondence:

Marcos Borato Viana

Faculdade de Medicina - Departamento de Pediatria

Universidade Federal de Minas Gerais

Av. Alfredo Balena, 190/267

CEP 30130-100 - Belo Horizonte, MG - Brazil

Tel.: +55 (31) 3409.9772

Fax: +55 (31) 3409.9770

E-mail: vianamb@gmail.com 